\title{
GRAIN SIZE DISTRIBUTION AND HEAT CONDUCTIVITY OF COPPER PROCESSED BY EQUAL CHANNEL ANGULAR PRESSING
}

\author{
O.V. Gendelman ${ }^{1}$, M. Shapiro ${ }^{1}$, Y. Estrin ${ }^{2}$, and R.J. Hellmig ${ }^{2}$ \\ ${ }^{1}$ Faculty of Mechanical Engineering, Technion - Israel Institute of \\ Technology, Haifa, Israel \\ ${ }^{2}$ Institute of Material Science and Engineering, Technical University of \\ Clausthal, Clausthal-Zellerfeld, Germany
}

\begin{abstract}
We report the results of measurements of the grain size distribution function and the thermal conductivity of ultrafine-grained copper produced by equal channel angular pressing (ECAP), with special attention to the evolution of these quantities with the number of pressing cycles. To explain the experimental findings, the equilibrium grain size distribution function (GSDF) evolving during ECAP has been calculated on the basis of a simplified theoretical model. The model involves a single unknown physical parameter - the most probable grain size. With this parameter fitted to the experimental data the calculated GSDF fairly closely reproduces the experimental data. A model for thermal conductivity of ECAP processed copper has been proposed, which relates thermal conductivity to the GSDF parameters to the coefficient of electron reflection at grain boundaries.
\end{abstract}

\section{Introduction}

Over the last decade, equal channel angular pressing (ECAP) has become a popular method for producing bulk ultrafine grained materials [1-3]. It is performed via repetitive pressing of a billet through a die having two intersecting channels of the same cross-section without changing the billet's cross-sectional dimensions. Therefore, extremely high shear strains can be imparted on a workpiece [4, 5], leading to grain refinement down to sub-micrometer scale.

Grain refinement by ECAP may be considered as a result of the formation and evolution of dislocation cell structure, accompanied with growth of the disorientation angles between the dislocation cells. The evolution of the grain size distribution with increasing equivalent strain has been determined using transmission electron microscopy (TEM) [1]. For a specimen that underwent a single ECAP pass, the dislocation cell disorientation distribution was determined [3]. The distribution of the grain size $s$ of submicron grained copper was studied in $[1,4,6]$ for samples obtained by high pressure torsion. A maximum of the grain size distribution function (GSDF)

$(s)$ reported there was at the grain size of about $107 \mathrm{~nm}$ and the distribution function was claimed to be log-normal. No attempt was made to either substantiate or rationalize this claim or predict the shape of the GSDF from modelling considerations.

The grain structure in ECAP processed metallic materials as observed by transmission electron microscopy is characterized by elongated grains [7-10]. The average grain size decreases during strain accumulation as a result of the increasing number of ECAP passes. The shape of the grains varies as well, resulting in elongated grains $[9,10]$. After about 8-10 passes (and sometimes earlier) the average grain size reaches saturation. In some cases a non monotonic variation of the average grain size with the number of passes was observed [11]. The GSDF under severe plastic deformations is commonly claimed to be close to log-normal [7]. It should be mentioned that, despite strong anisotropy of the grains, their distribution is usually 
characterized by a single directly measurable size parameter $s$. The GSDF evolution occurring during ECAP is important as it underlies the concomitant changes in mechanical properties and thermal conductivity with the number of deformation cycles.

Physically it is clear that thermal conductivity should exhibit an inverse dependence on the dislocation density $t$, because these dislocations constitute thermal resistances to the heat flow. This is also true for electric resistance of copper and gold nanowires $[12,13]$ and dense ceramic materials [14]. These investigations had demonstrated that the deviations of electric and thermal conductivities from the respective bulk properties are primarily related to electron scattering at grain boundary dislocations. The authors are not aware of independent experimental results on this issue.

This communication is aimed at developing a theoretical model for grain size distribution in copper specimens severely deformed by ECAP. The model is applied to the experimental GSDF measured by the authors [11] that show a non monotonic variation of the size-distribution function, and in particular, mean grain size of a copper specimen with the number of ECAP cycles. The model is further used to rationalize the non monotonic behaviour of the thermal conductivity of ECAPprocessed copper observed in the present study.

\section{EXPERIMENTAL}

\section{ECAP processing and grain size distribution measurements}

Workpieces for ECAP were cut from a coarse-grained polycrystalline $99.95 \%$ purity copper having an initial grain size of $30 \mu \mathrm{m}$ (after annealing of the as received material for 2 hours at $450^{\circ} \mathrm{C}$ ). ECAP route $\mathrm{C}$, in which the specimen is rotated by $180^{\circ}$ about the extrusion direction prior to each subsequent pass, was applied. The split design ECAP die with cross-sectional channel dimensions of $12 \mathrm{~mm} \times 12 \mathrm{~mm}$ and a corner angle of $20^{\circ}$ used was described in [11]. The pressing speed was 8 $\mathrm{mm} / \mathrm{min}$. A most significant grain refinement occurred already after the first ECAP pass; the subsequent passes produced only moderate further grain refinement saturating after several passes. Size distributions were determined by analyzing TEM micrographs. The apparent dislocation cell size was determined from bright field TEM micrographs using the ImageC image analysis program. The areas of the cells measured in two directions were related to a circle with the same area. For each sample, about 150 dislocation cells were evaluated. The resulting distributions, cf. Fig. 1, were fit by a log-normal distribution function [11]. 


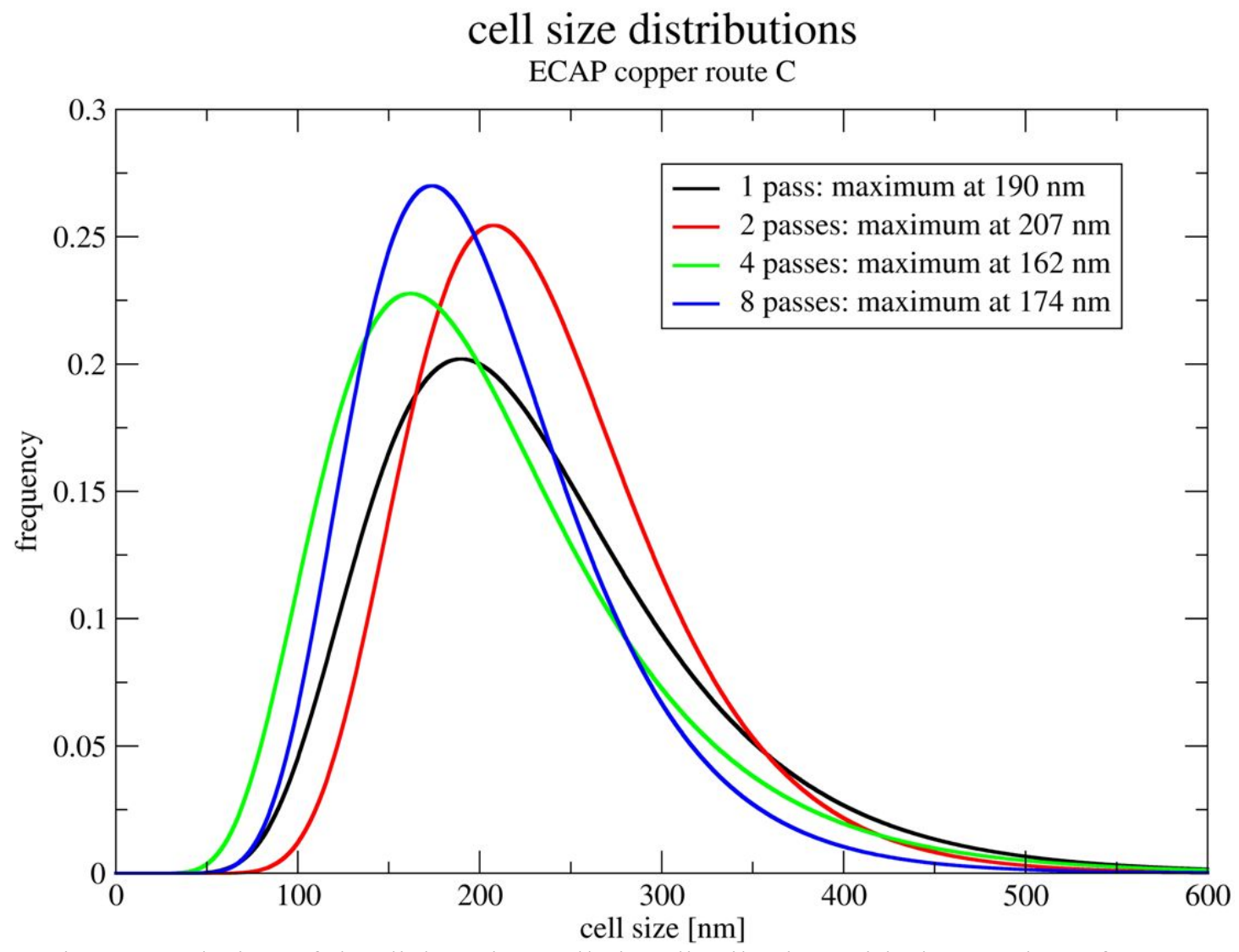

Fig. 1. Evolution of the dislocation cell size distribution with the number of ECAP passes measured by TEM. Note the oscillatory character of this evolution.

\section{Measurement of thermal conductivity}

We used the stationary method of thermal conductivity measurements [10] in which a copper specimen and a reference (Macor) specimen of known thermal conductivity, $k_{r e f}$, were subjected to the same unidirectional heat flux (see Fig. 2). The two specimens were placed between the heated and the cooled plates, which produced the heat flux. Thermal resistance between the two specimens and between the specimens and the plates was kept at a minimum by polishing the specimens and using thermally conducting graphite lubrication paste. Measurements of temperature differences along the copper specimen $\left(T_{1}-T_{2}\right)$ and the reference specimen $\left(T_{3}-T_{4}\right)$, along with the expression yield the thermal conductivity $k$ of the copper specimen.

$$
k \frac{T_{1}-T_{2}}{l}=k_{\text {ref }} \frac{T_{3}-T_{4}}{b},
$$

here $l$ and $b$ are the distances between the thermocouples in the powder bed and in the specimen, respectively. Temperatures were measured using Chromel-Alumel thermocouples. Our estimates showed that the effects of radiation and other mechanisms interfering with the measurements on the data for $k$ were negligible (below $0.01 \mathrm{~W} / \mathrm{mK}$ ). The overall measurement accuracy, including uncertainties in placing thermocouples within the specimens, deviations of the temperature field from unidirectional heat flow and errors induced by thermocouples, did not exceed $8 \%$, based on the comparison of the results with literature data for conductivities measured for several standard materials (such as stainless steel 304). 


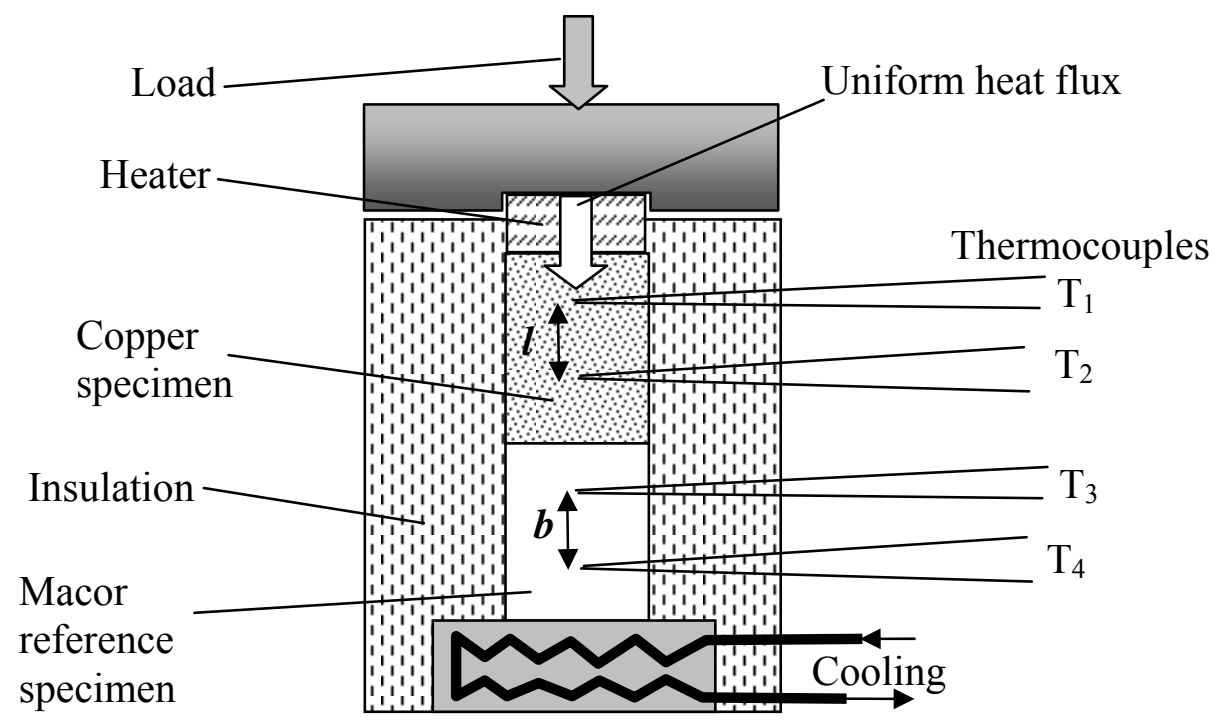

Fig. 2. Schematic of the thermal conductivity measurement cell: the stationary method

Thermal conductivities of four specimens were measured after first, second, fourth and eighth ECAP passes, sixteen specimens having been tested altogether. Thermal conductivity of unprocessed copper was taken from literature data. Figure 3 depicts the experimental measurement results, showing nonmonotonic oscillatory-like behavior with a tendency towards a constant value with increasing number of passes. 


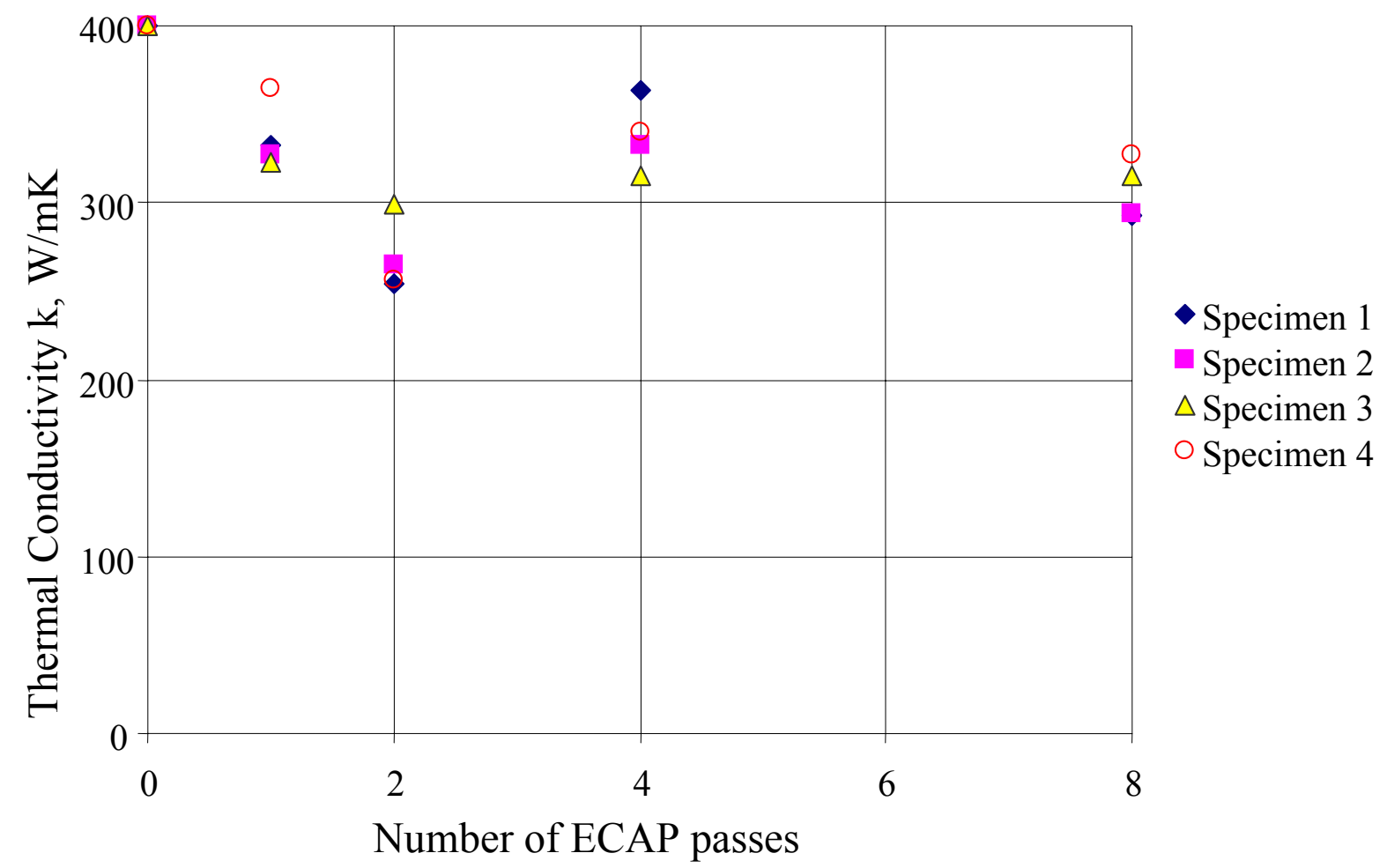

Fig. 3. Thermal conductivity of copper specimens the number of ECAP passes

\section{THEORETICAL GRAIN SIZE DISTRIBUTION}

Grain refinement during severe plastic deformation is associated with the formation and evolution of dislocation cell/subgrain boundaries with subsequent growth of the disorientation angle between the neighboring cells/subgrains [18]. As a result, a cellular dislocation structure develops with the cell (grain) walls containing a major portion of the dislocation density, as opposed to the cell interior where the dislocation density is significantly lower. Several mechanisms of formation, motion and annihilation of the dislocations within the cell interior and cell walls are known $[16,17]$, which together govern the emerging GSDF and its evolution with the imposed strain. In what follows, we shall not distinguish between the dislocation cells/subgrains and grains, the former being treated as the smallest structural unit. As the average dislocation cell/subgrain size scales with the inverse of the square root of the total dislocation density $\rho_{t}[16,17]$, the increase of $\rho_{t}$ with strain promotes grain refinement during straining. We shall also assume that concurrent in situ cell/subgrain growth acts against this trend.

Specific mechanisms governing the evolution of the dislocation density in cell interiors, $c$, and in cell walls $w$, include (i) generation of dislocations due to activation of Frank-Read sources at the cell walls (ii) the motion (transport) of cellinterior dislocations and their 'entrapping' in the walls, and (iii) annihilation due to cross-slip or climb $[16,17]$. The rate of accumulation of cell interior dislocations is considered to be inversely proportional to their mean free path, which is proportional to the average grain size $s_{m}$. In turn, as mentioned above, the average grain size is 
assumed to be inversely proportional to the total dislocation density $\rho_{t}$ (the weighted average of $\rho_{c}$ and $\left.\rho_{w}\right)$, i.e., $s_{m} \propto\left(\rho_{t}\right)^{-1 / 2}$.

Summarizing the situation with theory, one can note that neither does the current status of modeling of strain hardening account for the grain size distribution nor does it provide means for rationalizing the effect of strain rate on this function. Therefore at this stage it is reasonable to devise a simplest model allowing for at least a qualitative description of the size distribution of the grains. We will adopt the following assumptions concerning every ECAP pass:

Each grain is characterized by a single geometric parameter - a characteristic size s. In fact the grains have complicated shapes and are by no means isotropic $[1,5]$. Besides, according to experimental evidence grains in strongly deformed copper [17] and ultrafine grained specimens of other metals (e.g. Ni [5], Al [10], etc.) are elongated in the direction of shear. Also the determination of grain size depends on the boundary identification procedure implicit in the TEM-based measurement of $s$. Still, in order to gain insight in the GSDF we will describe the grain geometry by a single parameter $s$, which (keeping in mind the qualitative nature of such description) can be associated with the grain thickness in the direction perpendicular to shear.

We describe the grain refinement, in a somewhat schematic way, as a result of two competing processes: grain division and concurrent grain growth. For the sake of simplicity, we assume that a grain is subdivided into two grains of smaller size. The probability $p_{d i v}(s)$ of such division process depends on the characteristic grain size $s$.

To simplify things further, we assume that the characteristic sizes of the resulting two grains are equal to half of the characteristic size of the original grain. This assumption supports volume conservation in the process of subdivision only if the grain volume scales linearly with $s$. We further assume that fusion, or conglomeration, of two grains of characteristic sizes $s_{1}$ and $s_{2}$ to a grain with characteristic size $s=s_{1}+s_{2}$ is governed by probability density $p_{c o n}\left(s_{1}, s_{2}\right)$ Under the above assumptions, a kinetic equation for the evolution of the grain size distribution function during an ECAP pass can be formulated:

$$
\begin{aligned}
& \frac{\partial f(s, t)}{\partial t}=-p_{d i v}(s) f(s)+2 p_{d i v}(2 s) f(2 s) \\
& +\int_{0}^{s} p_{c o n}(z, s-z) f(z) f(s-z) d z-f(s) \int_{0}^{\infty} p_{c o n}(z, s) f(z) d z
\end{aligned}
$$

where $f(\mathrm{~s}, \mathrm{t})$ is the GSDF at a given moment of time $t$ within the pass (the $t$ dependence of $f$ on the right-hand side of Equation (2) is suppressed for brevity). The first term on the right-hand side describes the division rate (per unit size range) of the grains having size $s$. The second term corresponds to the rate of increase of the number of grains of size $s$ due to division of larger grains. The third term describes the gain in the number of grains of size $s$ due to conglomeration of smaller grains. Finally, the last term is related to the loss of grains of size $s$ due to their conglomeration with other grains. The GSDF is to be normalized to satisfy the condition

$$
\int_{0}^{\infty} f(s) d s=1
$$

It is easy to verify that Equation (2) preserves this normalization. Indeed, integration of both sides of Equation (2) leads to the following result: 


$$
\begin{aligned}
& \left.\int_{0}^{\infty} \frac{\partial f(s, t)}{\partial t} d s=\frac{\partial}{\partial t} \int_{0}^{\infty} f(s) d s=\int_{0}^{\infty}\left\{-p_{d i v}(s) f(s)+2 p_{d i v}(2 s) f(2 s)\right)\right\} d s \\
& +\int_{0}^{\infty}\left\{\int_{0}^{s} p_{c o n}(z, s-z) f(z) f(s-z) d z-f(s) \int_{0}^{\infty} p_{c o n}(z, s) f(z) d z\right\} d s= \\
& =-\int_{0}^{\infty} p_{d i v}(s) f(s) d s+\int_{0}^{\infty} p_{d i v}(2 s) f(2 s) d(2 s)+ \\
& +\int_{0}^{\infty} \int_{0}^{s} p_{c o n}(z, s-z) f(z) f(s-z) d z d s-\int_{0}^{\infty} \int_{0}^{\infty} p_{c o n}(s, z) f(z) f(s) d z d s=0
\end{aligned}
$$

An experimental ECAP pass is long enough to assume that at every pass an equilibrium distribution is achieved. This equilibrium distribution $f_{\mathrm{e}}(\mathrm{s})$ is described by the following equation:

$$
\begin{aligned}
& 0=-p_{d i v}(s) f_{e}(s)+2 p_{d i v}(2 s) f_{e}(2 s) \\
& +\int_{0}^{s} p_{c o n}(z, s-z) f_{e}(z) f_{e}(s-z) d z-f_{e}(s) \int_{0}^{\infty} p_{c o n}(z, s) f_{e}(z) d z
\end{aligned}
$$

which should be solved for given functions $p_{d i v}(s)$ and $p_{c o n}\left(s_{1}, s_{2}\right)$.

Equation (4) is a strongly nonlinear integral equation, hardly solvable analytically. For simplicity we assume that only grains of equal sizes may conglomerate. This implies that the probability of conglomeration is given by:

$$
p_{\text {con }}\left(s_{1}, s_{2}\right)=g\left(s_{1}+s_{2}\right) \delta\left(s_{1}-s_{2}\right)
$$

where $\delta$ is Dirac's delta-function. Substituting (5) into (4), one gets

$$
0=-p_{d i v}(s) \rho_{e}(s)+2 p_{d i v}(2 s) f_{e}(2 s)+\frac{1}{2} g(s) f_{e}^{2}(s / 2)-g(2 s) f_{e}^{2}(s)
$$

It is easily seen that any function $\rho_{e}(s)$ that satisfies the equation

$$
\frac{f_{e}(2 s)}{f_{e}^{2}(s)}=\frac{g(2 s)}{2 p_{d i v}(2 s)}
$$

also satisfies Equation (6). Physically, equation (6) expresses equilibrium between division of grains of size $s$ into two groups of grains of size $s / 2$ and the reverse recombination (conglomeration) process. It can be observed that for any function $\rho_{e}(s)$ which satisfies Equation (10), the product $f_{\mathrm{e}}(\mathrm{s}) \exp (k s)$, where $k$ is an arbitrary constant, also satisfies this equation. Therefore we shall search for a solution in the form

$$
f_{e}(s)=\phi(s) \exp (-\sigma s)
$$

where $f(s)$ satisfies Equation (7) and $\sigma$ can be determined from the normalization condition, Equation (3).

In order to find $(s)$, detailed information concerning $p_{d i v}(s)$ and $g(s)$ is required. The real process of grain refinement is complicated and includes essential changes of the grain shapes. In our coarse model we assume that, as division is a result of formation of dislocation walls due to reactions between grain interior dislocations, its probability density should be proportional to the number of nucleation sites, $\rho_{c} s^{2}$, and to $n / \tau$, where $n \propto \rho_{c} l^{2}=$ const is the number of dislocations located at a characteristic distance $l \cong 1 / \sqrt{\rho_{c}}$ from a potential nucleation site and $\tau=l / v=l \rho_{c} b / \dot{\gamma}$ is a characteristic time it takes these dislocations to travel to a 
nucleation site. The resulting probability of cell/grain division per unit time can be written in the form

$$
p_{d i v}=A \dot{\gamma} \sqrt{\rho_{c}} s^{2} / b
$$

where $A$ is a non-dimensional constant of the order of unity.

In the above expression the dislocation velocity $v$ was expressed in terms of the shear rate $\dot{\gamma}$ (assumed to be the same within the grains and in the grain boundaries) via the Orowan relation.

As stated before, we also assume that two neighboring grains with size $s$ may conglomerate to a bigger grain with size $2 s$, the probability of such an event being $g(s)$. As a qualitative estimate of the probability of conglomeration we assume that it is controlled by cell/grain boundary motion driven by the energy stored in the boundaries. Accordingly, the probability of grain size doubling ('conglomeration') will be inversely proportional to the characteristic time $s /(m p)$, where $m$ is the boundary mobility and $p$ is the driving force given by

$$
p \propto E / s,
$$

here

$$
E \propto G b^{2} w \rho_{w} \propto G b^{3} \rho_{w}
$$

is the energy per unit area of the grain boundary, $G$ is the shear modulus and $w$ is the boundary thickness, typically of the order of $3 b$. In Equation (11) use was made of the fact that the density of dislocations in the walls is by far larger than that of cellinterior dislocations. This leads to the following expression for the probability of conglomeration:

$$
g(s)=B \frac{m G b^{3} \rho_{w}}{s^{2}},
$$

where $B$ is a constant of the order of unity.

Needless to say, the crude model formulated above neglects many essential physical details of the process. Possible changes of shape of the grains accompanying the division and conglomeration processes, and their relaxation after these processes are not taken into account. As stated above, a grain is characterized by a single parameter - its size. As suggested by Equations (9) and (12), the probability $p_{d i v}(s)$ behaves as $s^{2}$, while $g(s)$ should decrease with growing $s$ (obeying an inverse square law), yielding

$$
\frac{\phi(2 s)}{\phi^{2}(s)}=\frac{g(2 s)}{2 p_{d i v}(2 s)} \sim s^{-4}
$$

More generally, it is reasonable to assume that

$$
\frac{\phi(2 s)}{\phi^{2}(s)}=\frac{g(2 s)}{2 p_{d i v}(2 s)} \sim s^{-v}, v>0 .
$$

From Equations (8) and (14) it follows that the equilibrium GSDF for deforming copper may be approximated by:

$$
f_{e}(s)=\frac{\sigma^{v+1}}{\Gamma(v+1)} s^{v} \exp (-\sigma s) .
$$

Calibration of this function to provide adequate fit of the GSDF to the experimental data is required, as explained below.

The GSDF given by Equation (15) depends on two parameters, and $\sigma$, one of which may be determined from the normalization condition. Rather than using the latter condition we will determine these two parameters directly from the experimental data for every measured GSDF using the size, $s_{\max }$, corresponding to the 
maximum of the distribution and the value of the GSDF at the maximum. These two quantities are related to the GSDF parameters as follows:

$$
\begin{aligned}
& s_{\max }=\frac{v}{\sigma} \\
& f_{e}\left(s_{\max }\right) s_{\max }=\frac{v(v / e)^{v}}{\Gamma(v+1)}
\end{aligned}
$$

The parameters of analytical approximations for the GSDFs for different numbers of ECAP passes presented in Fig. 1 are compiled in Table 1. The example of fitting the GSDF predicted by Equation (15) to raw experimental data is presented in Fig. 4.

Table 1. Fitting parameters of the GSDF curves computed from the experimental data (Fig. 1) with the help of Equations (15) and (16).

\begin{tabular}{|c|c|c|c|}
\hline Number of cycles & $\sigma, \mathrm{nm}^{-1}$ & $v$ & $\mathrm{~s}_{\max }, \mathrm{nm}$ \\
\hline 1 & 0.02 & 3.79 & 190 \\
\hline 2 & 0.035 & 7.167 & 207 \\
\hline 4 & 0.022 & 3.5 & 162 \\
\hline 8 & 0.033 & 5.71 & 174 \\
\hline
\end{tabular}
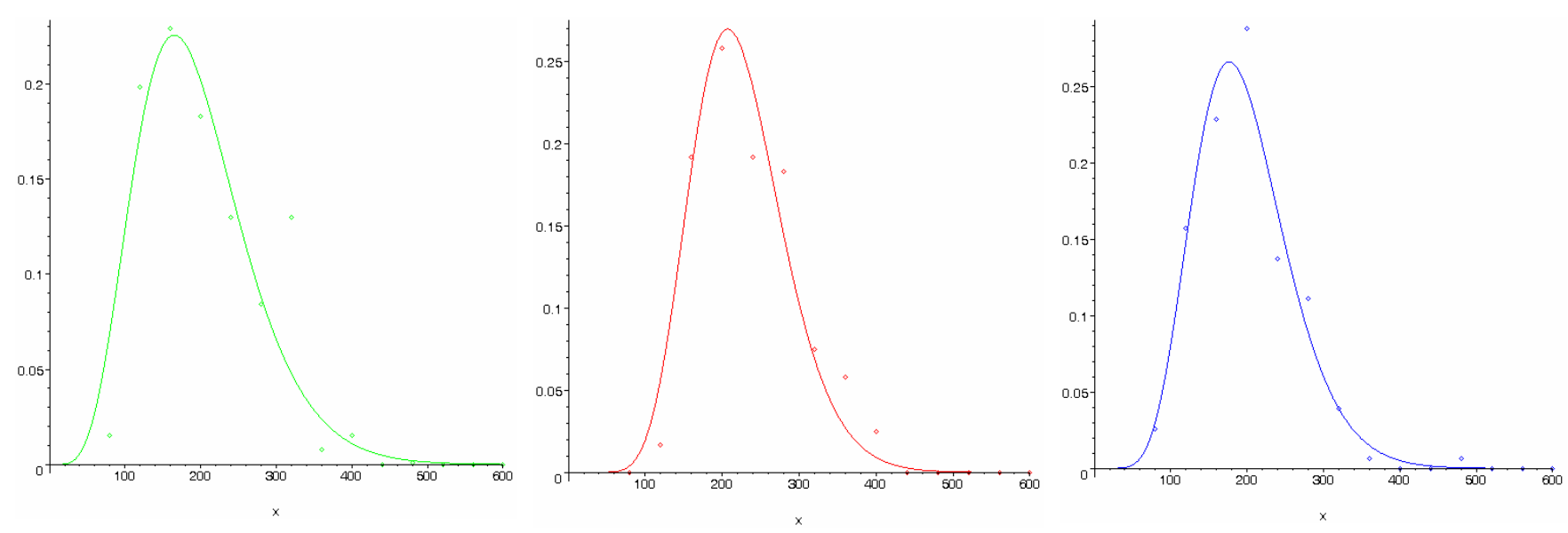

Fig. 4. Normalized GSDF computed from Equations (15) and (16) (solid line) versus experimental points obtained after two deformation cycles (left), four cycles (middle) and eight cycles (right). (Experimental data from Fig. 1 were used.)

The fit with the experimental plots is fairly good, despite crude approximations used in deriving Equation (14). Distribution function (14) is not lognormal, contrary to statements in $[1,2,5]$. It should be mentioned that the experimental data can also be reasonably well fitted with the log-normal distribution, as is often done in literature without further justification $[1,2,5]$. However, the advantage of the GSDF given by Equation (15) is that it can be justified on the basis of the model proposed. Besides, it should be mentioned that the value of $v$ obtained from the experimental data in the majority of cases is fairly close to the value of 4 proposed above, despite the mentioned crudeness of the model suggested.

Similar estimation of the GSDF may be done on the basis of volume conservation, i.e. if the grain volume is used instead of the linear size parameter $s$. Such simulation has been performed and the matching of the GSDF led to much less accord with the experimental data; namely, the function obtained failed to predict the character of GSDF behavior in the region of small grain sizes. This observation seems 
to support the proposition that the division and conglomeration processes may be considered as essentially one-dimensional ones.

\section{THEORETICAL MODEL FOR HEAT CONDUCTIVITY}

No data on the effect of ECAP on the thermal conductivity of ductile polycrystalline materials was reported so far. Therefore the purpose of this section of the paper is to provide a basic explanation of the oscillatory type behavior of $k$ observed, cf. Figure 2.

Information concerning the dependence of grain size on the number of cycles of deformation in bulk nanostructured materials is limited. Generally, generation of dislocations is accompanied with a decrease of the average grain size $s_{\text {ave }}$ and the concomitant increase of the resolved shear stress [12], which is a generally known hardening trend. However, in some cases a non monotonic evolution of the average grain size was recorded, as in pure aluminum specimens deformed by ECAP [6]. Namely, both the value of the average grain size and the character of its evolution were found to depend on geometric peculiarities of the ECAP process, giving qualitatively different results for different planes and ECAP routes. This non monotonic evolution of $s_{\text {ave }}$ resembles the trend observed for grain size distributions in Figure 1.

For the sake of qualitative description, let us consider a one-dimensional chain of $N$ grains of equal size $l$, total length $L=N l$ and cross-sectional area $S$. The overall thermal resistance of this chain is

$$
R=\frac{L}{S \kappa_{\text {bulk }}}+N R_{\text {disl }}=\frac{L}{S \kappa_{\text {bulk }}}+\frac{L}{l} R_{\text {disl }}
$$

where $k_{b u l k}$ is the bulk heat conductivity and $R_{\text {disl }}$ is the thermal resistance of dislocations. $R_{\text {disl }}$ may be evaluated using the fact that for electron gas electrical and thermal conductivities are proportional to each other, cf. the Wiedemann-Franz law. Therefore it is possible to take advantage of known estimates of grain boundary electrical resistance $[13,15]$ :

$$
R_{\text {disl }}=\frac{4 \lambda}{3 A \kappa_{\text {bulk }}(1-r)},
$$

where $\lambda$ is the electron mean free path, $A$ is the contact area and $r$ is the electron reflectivity. The latter term takes into account the fact that some electrons are reflected from the grain boundary dislocations and therefore do not participate in the transfer process.

Geometry of grain surfaces is complicated and in order to take into account that the contact is only partial we adopt $A=S / 2$. Combining (17) and (18), one obtains:

$$
R=\frac{L}{S \kappa_{\text {bulk }}}\left(1+\frac{2 \lambda}{3 l(1-r)}\right)
$$

Introducing the experimentally measured effective thermal conductivity $\kappa_{\exp }$ via the equation

$$
R=\frac{L}{S \kappa_{\exp }}
$$

one finally gets

$$
\kappa_{\exp }=\frac{\kappa_{\text {bulk }}}{1+\frac{2 \lambda}{3 l(1-r)}}
$$


In order to evaluate the reflectivity, a reasonable estimate of $l$ needs to be substituted into Equation (21). Table 2 contains the estimation based on the average grain size $s_{\text {ave }}$ corresponding to the GSDF for each particular case. Values used are $\kappa_{b u l}=400 \mathrm{~W} / \mathrm{m} / \mathrm{K}, \lambda=39 \mathrm{~nm}([7], T=300 \mathrm{~K})$.

We are not aware of experimental data concerning the electron reflectivity in submicron grains in copper. The only data for comparison available are related to gold [13], which has chemical and physical properties rather similar to copper. For nanostructured gold the reflectivity was found to be in the range between 0.2 and 0.8 , which is consistent with the results for $\mathrm{Cu}$ presented above.

It should be mentioned that in the cases of 2 and 8 ECAP passes the values of the reflectivity are significantly higher than in the other two cases. One can speculate that these higher values correspond to more narrow size distribution functions, since packing conditions for such distributions are less favorable and hence the matching between boundaries of neighboring grains is worse. This conclusion is supported by the data on the standard deviation calculated from the GSDF, Equation (16), for each case - larger values of the reflection coefficient are correlated with smaller values of the standard deviation. The results of correlation analysis are presented in Table 2. The data available are restricted and do not allow quantitative analysis, but qualitatively, the correlation is obvious.

Table 2 Correlation between the "relative width" of the distribution function and electron reflection coefficient for various numbers of ECAP passes.

\begin{tabular}{|c|c|c|c|c|c|}
\hline $\begin{array}{c}\text { Number } \\
\text { of } \\
\text { cycles }\end{array}$ & $\begin{array}{c}\text { Thermal } \\
\text { conductivity } \\
\mathrm{W} / \mathrm{mK}\end{array}$ & $\begin{array}{c}\text { Average } \\
\text { grain size } \\
<l>\mathrm{nm}\end{array}$ & $\begin{array}{c}\text { Standard } \\
\text { deviation, } \\
\mathrm{nm}\end{array}$ & $\begin{array}{c}\text { Relative width of the } \\
\text { distribution } \\
\text { (Standard } \\
\text { deviation/average size) }\end{array}$ & $\begin{array}{c}\text { Electron } \\
\text { reflectivity } \\
\mathrm{r}\left(s_{\text {ave }}\right)\end{array}$ \\
\hline 1 & 340 & 240 & 130.38 & 0.541 & 0.38 \\
\hline 2 & 280 & 233 & 90.87 & 0.390 & 0.73 \\
\hline 4 & 330 & 204 & 115.9 & 0.563 & 0.39 \\
\hline 8 & 310 & 203 & 89.12 & 0.44 & 0.56 \\
\hline
\end{tabular}

\section{Summary and concluding remarks}

The measured grain size distribution function in ECAP processing was shown to exhibit a non monotonic behavior with increasing number of ECAP passes. A simple and relatively crude model based on the grain/cell population balance equation was used to calculate the grain size distribution function for ECAP processing. Calibration of the model requires a basic length scale as a fit parameter, and the most probable grain size was used for this purpose. With this single fit parameter, the model fairly accurately reproduces the grain size distribution within the copper specimens, which is independent of the ECAP parameters. This suggests that the size distribution achieved during ECAP passes has a general form, characterized by a single length parameter, in which the influence of the ECAP processing is manifested.

The model for thermal conductivity of the copper specimens proposed allows rationalizing the dependence of this property in terms of the electronic reflectivity. This quantity, extracted form the thermal conductivity model, is shown to depend inversely on the width of the grain size distribution, as expressed in terms of the standard deviation to average grain size ratio of the GSDF. 


\section{REFERENCES}

1. Valiev R.Z., Islamgaliev R.K., Alexandrov I.V., Bulk Nanostructured Materials from Severe Plastic Deformation, Progress in Material Science, 45 (2000) pp. 103-189.

2. http://www.nanospd.org

3. Estrin, Y., Effects of Severe Plastic Deformation: Mechanical properties and Beyond, Mater. Science Forum503-504 (2006) 91-98.

4. Islamgaliev S.K., Chmelik F., Kuzel R., Thermal Stability of Submicron Grained Copper and Nickel, Mater. Sci. Eng. A237 (1997) 43-51.

5. Segal, V.M.: Mater. Sci. Eng. A197 (1995) 157-166.

6. Gerstman V. Yu., Birringer R., Valiev RZ. and Gleiter H., Scripta Metall Mater., 30 (1994), 229-234.

7. A.P.Zhilyaev, B.-K.Kim, J.A.Szpunar, M.D.Baro, T.G.Langdon, The Microstructural Characteristics of Ultrafine - Grained Nickel, Materials Science and Engineering, A391, (2005) 377-389.

8. Y. Iwahashi, Z. Horita, M. Nemoto and T.G. Langdon, The Process of Grain Refinement in Equal - Channel Angular Pressing, Acta Mater., 46 (1998) 33173331.

9. K. Nakashima, Z. Horita, M. Nemoto and T.G. Langdon. Influence of channel angle on the development of ultrafine grains in equal channel angular pressing. Acta Mater. 46 (1988) 1589-1599.

10. P.J. Apps, J.R. Bowen, P.B. Prangnell, The effect of second-phase particles on the severe deformation of aluminum alloys during Equal Channel Angular Extrusion. Proceedings of NanoSPD2 Conference, Vienna, 9-13 December 2002, M. J. Zehetbauer and R.Z. Valiev, Eds., Wiley-VCH, Weinheim, 2004, pp. 138144.

11. R.J. Hellmig, S.C. Baik, M.H. Seo, H.S. Kim, Y. Estrin "Effect of equal channel angular pressing on microstructure and tensile properties of pure copper" Proceedings of the first Russian-French symposium "physics and mechanics of large plastic strains", V.Rybin and C. Teodosiu (eds.), St. Petersburg 2003, published in: Problems of materials science 1 (33) 2003, pp. 168-174.

12. W.Wu, A.H.Brongersma, M. Van Hove and K.Maex, Influence of Surface and Grain - Boundary Scattering on the Resistivity of Copper in Reduced Dimensions, Applied Physics Letters, 84 (2004) 2838-2840.

13. A. Bietsch, B. Michel, Size and Grain - Boundary Effects of a Gold Nanowire Measured by Conducting Atomic Force Microscopy, Applied Physics Letters, 80 (2002), 3346-3348.

14. E. Litovsky, M. Shapiro, Gas pressure and temperature dependences of thermal conductivity of porous ceramic materials: Part 1. Refractories and ceramics with porosity below 30\%, J. Am. Ceram. Soc., 7 (1992) 3425-3439.

15. Y.V.Sharvin, JETP 21 (1965), 655

16. S. C. Baik, Y. Estrin, H. S. Kim, R. J. Hellmig. Dislocation density-based modeling of deformation behavior of aluminum under equal channel angular pressing. Mater. Sci. Eng. A351 (2003) 86-97.

17. Y. Estrin, L.S. Toth, A. Molinari and Y. Brechet. A dislocation-based model for all hardening stages in large strain deformation. Acta Mater 46, 5509 - 22 (1998)

18. T.T. Lamark, R.J. Hellmig, Y. Estrin. Mechanical Properties of ECAP processed magnesium alloy AS21X. Material Scientific Forum Vol 503-504, pages 889-894 Trans Tech Publications Switzerland (2006). 